$5^{\text {th }}$ International Conference on Research in

Behavioral and Social Science

Spain | Barcelona | December 7-9, 2018

\title{
Shape Preference and the Gender Differences
}

\author{
Yasuto Okamura \\ Graduate School of Psychology, Otemon Gakuin University, Japan
}

\begin{abstract}
Previous studies have reported that individuals prefer curved (e.g., round) objects to angular (e.g., square) ones and that curved shapes are preferred more by females and angular shapes more by males. The present study replicated these findings. The study included 100 male and 100 female participants, aged 20-50 years. Participants were asked which shape (round or square) was preferable. A univariate chi-square test revealed that the round shape was generally preferred $(57 \%)\left(\chi^{2}(1)=\right.$ $3.92, p=.048, \phi=.14$ ). Moreover, a 2 (male/female gender) $\times 2$ (round/square shape) chi-square test showed that the percentage who preferred the round shape was higher for females (57\%) than males (43\%) whereas the percentage preferring the square shape was higher for males (59\%) than females (41\%) $\left.\chi^{2}(1)=4.59, p=.032, \phi=.15\right)$. This gender difference in shape preference suggests a fundamental difference between males and females in perceptual style.
\end{abstract}

Keywords: shape, preference, gender differences 